\title{
Intermediate-Term Memory for Site-Specific Sensitization in Aplysia Is Maintained by Persistent Activation of Protein Kinase C
}

\author{
Michael A. Sutton, ${ }^{1,2}$ Martha W. Bagnall, ${ }^{2}$ Shiv K. Sharma, ${ }^{2}$ Justin Shobe, ${ }^{2}$ and Thomas J. Carew ${ }^{2}$ \\ ${ }^{1}$ Interdepartmental Neuroscience Program, Yale University, New Haven, Connecticut 06520-8074, and 2Department of Neurobiology and Behavior, Center \\ for the Neurobiology of Learning and Memory, University of California Irvine, Irvine, California 92697-4550
}

\begin{abstract}
Recent studies of long-term synaptic plasticity and long-term memory have demonstrated that the same functional endpoint, such as long-term potentiation, can be induced through distinct signaling pathways engaged by different patterns of stimulation. A critical question raised by these studies is whether different induction pathways either converge onto a common molecular mechanism or engage different molecular cascades for the maintenance of long-term plasticity. We directly examined this issue in the context of memory for sensitization in the marine mollusk Aplysia. In this system, training with a single tail shock normally induces short-term memory $(<30$ $\mathrm{min}$ ) for sensitization of tail-elicited siphon withdrawal, whereas repeated spaced shocks induce both intermediate-term memory (ITM) ( $>90 \mathrm{~min}$ ) and long-term memory ( $>24 \mathrm{hr}$ ). We now show that a single tail shock can also induce ITM that is expressed selectively at the trained site (site-specific ITM). Although phenotypically similar to the form of ITM induced by repeated trials, the mechanisms by which site-specific ITM is induced and maintained are distinct. Unlike repeated-trial ITM, site-specific ITM requires neither protein synthesis nor PKA activity for induction or maintenance. Rather, the induction of site-specific ITM requires calpain-dependent proteolysis of activated PKC, yielding a persistently active PKC catalytic fragment (PKM) that also serves to maintain the memory in the intermediateterm temporal domain. Thus, two unique forms of ITM that have different induction requirements also use distinct molecular mechanisms for their maintenance.
\end{abstract}

Key words: PKA; PKM; synaptic facilitation; learning; calpain; protein synthesis; proteolysis

\section{Introduction}

Although it is well established that distinct molecular mechanisms underlie different phases of memory and synaptic plasticity, recent studies have shown that multiple forms of synaptic plasticity with distinct molecular requirements can exist within the same temporal phase (Kang et al., 1997; Oliet et al., 1997; Bailey et al., 2000; Sutton and Carew, 2000). These cellular observations raise an important behavioral question: In the maintenance of memory, do different induction pathways ultimately converge on the same molecular mechanism, or are unique mechanisms for maintenance used once memories are fully formed?

The marine mollusk Aplysia provides a useful experimental system to directly analyze this question. One form of learning in Aplysia that is particularly well understood is sensitization, an increase in responsiveness induced by a noxious stimulus (such as tail shock). Facilitation of sensory neuron (SN) to motor neu-

Received April 16, 2003; revised Jan. 28, 2004; accepted Jan. 28, 2004.

This work was supported by National Institute of Mental Health Grant R01 MH-14-1083 to T.J.C. We thank Adam Bristol and Angela Purcell for helpful comments on a previous version of this manuscript and Wayne Sossin for kindly providing antibodies and for thoughtful discussions on our data.

Correspondence should be addressed to Dr. Thomas J. Carew, Department of Neurobiology and Behavior, University of California Irvine, Irvine CA 92697-4550. E-mail: tcarew@uci.edu.

DOI:10.1523/JNEUROSCI.1134-03.2004

Copyright $\odot 2004$ Society for Neuroscience $\quad$ 0270-6474/04/243600-10\$15.00/0 ron $(\mathrm{MN})$ synapses induced by serotonin $(5-\mathrm{HT})$ is a widely used cellular model of sensitization in Aplysia and can be differentiated into at least three distinct temporal phases: short-term facilitation ( $<30 \mathrm{~min}$ ), intermediate-term facilitation (ITF) ( $>90 \mathrm{~min}$ ), and long-term facilitation (LTF) $(>24 \mathrm{hr})$. Intermediate-term (IT) and long-term phases of synaptic facilitation are not unitary but rather encompass multiple forms of plasticity mediated by distinct molecular mechanisms (Casadio et al., 1999; Bailey et al., 2000; Sutton and Carew, 2000; Sherff and Carew, 2002). For example, activity-independent ITF induced by repeated 5-HT pulses requires protein synthesis for its induction and persistent activation of protein kinase A (PKA) for its maintenance (Ghirardi et al., 1995; Mauelshagen et al., 1996; Müller and Carew, 1998; Sutton and Carew, 2000). In contrast, activitydependent ITF, induced by a single pulse of 5-HT coupled with SN activation (Bao et al., 1998; Sutton and Carew, 2000), does not require protein synthesis for induction and requires a persistent activation of protein kinase C (PKC) for its maintenance (Sutton and Carew, 2000). Finally, similar to activity-independent ITF, repeated tail shocks induce an intermediate phase of memory [intermediate-term memory (ITM)] for sensitization that requires protein synthesis for its induction and persistent PKA activity for its maintenance (Sutton et al., 2001).

The fact that two forms of ITF in Aplysia use distinct mechanisms for induction and maintenance raises the possibility that 
different patterns of behavioral experience might recruit distinct molecular mechanisms in the service of maintaining memory over the same IT temporal domain. We examined this issue by characterizing the molecular mechanisms underlying the induction and maintenance of an enhanced (site-specific) form of sensitization, which is revealed by eliciting reflex responses with tactile stimulation at the site of a previous tail shock (Walters, 1987). We found that the induction of site-specific ITM requires initial activation of PKC and that the maintenance of site-specific ITM requires persistent $\mathrm{PKC}$ activation, the latter derived from proteolytic generation of a persistently active PKC catalytic fragment (PKM). Our results illustrate a novel role for persistent PKC activation in maintaining intermediate-term memories and demonstrate that unique forms of memory can be maintained over the same time domain by distinct mechanisms.

\section{Materials and Methods}

Behavioral procedures. The reduced behavioral preparation used in these experiments has been described in detail previously (Sutton et al., 2001). Briefly, wild-caught Aplysia californica (250-400 gm; Marinus, Long Beach, CA) were anesthetized by injection of isotonic $\mathrm{MgCl}_{2}(\sim 100 \mathrm{ml} /$ 100 gm of body weight). The tail and mantle were surgically removed, with the ring ganglia, abdominal ganglion, and their peripheral connections remaining intact. To keep the tail and siphon suitably inflated throughout the experiment, each was continually perfused with cooled seawater (Instant Ocean, $15^{\circ} \mathrm{C}$; Aquarium Systems, Mentor, $\mathrm{OH}$ ). The siphon artery was cannulated with SILASTIC tubing (Dow Corning, Midland, MI) (inner diameter, 0.025 inches) and perfused at $\sim 5 \mathrm{ml} / \mathrm{min}$, and the tail was perfused at $\sim 0.5 \mathrm{ml} / \mathrm{min}$ through a 22 gauge needle inserted into the medial aspect of the tail. The tail and mantle were pinned to the Sylgard-coated floor of a chamber containing circulating tank seawater $\left(15^{\circ} \mathrm{C}\right)$, and the ring ganglia (composed of the cerebral and two paired pleural-pedal ganglia) were pinned ventral side up in a separate Sylgard-coated chamber and perfused continuously at room temperature $\left(20-22^{\circ} \mathrm{C}\right)$ with artificial seawater (ASW) containing the following (in mM): $460 \mathrm{NaCl}, 55 \mathrm{MgCl}_{2}, 11 \mathrm{CaCl}_{2} 10 \mathrm{KCl}$, and 10 Tris, $\mathrm{pH}$ 7.6. In all experiments, the ring ganglia (with pleural-pedal ganglia desheathed) were isolated in this separate subchamber and perfused independently (see Fig. 1A). The connections to the tail (via the P9 nerves) and abdominal ganglion (via the pleural-abdominal nerves) exited the subchamber through small slits that were sealed with petroleum jelly. Preparations were allowed at least $60 \mathrm{~min}$ to recover before pretest measurements.

Before training in all experiments, three pretests (intertrial interval, 15 min) were conducted to ensure a stable baseline of tail-elicited siphon withdrawal (T-SW) duration. In each of these tests, the right or left side of the tail ( $\sim 1 \mathrm{~cm}$ anterior from its tip, and midway between the lateral and medial margins) was stimulated with a brief water jet $(8 \mathrm{~Hz}, 0.5 \mathrm{sec}$ duration). The choice of the right or left side was counterbalanced in each experiment, and similar results were obtained for the right and left sides. For training, a single AC tail shock (1.5 sec duration) was delivered through a hand-held bipolar electrode to the test site, a different site ipsilateral to the test site $(0.5-1.0 \mathrm{~cm}$ posterior to the anterior margin of the tail), or a contralateral site directly opposite to the test site (see Fig. $1 A$ ). The nominal current across the electrode was $100 \mathrm{~mA}$, although much of this current is shunted by the seawater. Tests of T-SW after training (elicited in the same manner as the pretests described above) were conducted every 15 min beginning $15-30 \mathrm{~min}$ after training and continuing for 75-120 min.

All drug treatments were restricted to the ring ganglia subchamber. For experiments examining induction of sensitization, on completion of the third pretest, the ring ganglia were treated for 30-45 min before and during training, and the drug-vehicle was washed out immediately after the completion of training. For experiments examining the maintenance of sensitization, the ring ganglia were treated for 45 min beginning immediately after the $30 \mathrm{~min}$ post-test, and the drug-vehicle was washed out immediately after completing the 75 min post-test. In experiments examining protein synthesis requirements, the ring ganglia subchamber was perfused with the translation inhibitor emetine (Sigma, St. Louis, MO) for 40 min before pretests and throughout the duration of the experiment. All other drugs were obtained from Calbiochem (La Jolla, CA).

Western blotting. One set of experiments examined whether endogenous Aplysia PKC was a substrate for the calcium-activated protease calpain. In these experiments, whole CNSs (including the buccal, ring, and abdominal ganglia for each animal) were removed and desheathed in 50:50 ASW: $\mathrm{MgCl}_{2}$. After desheathing, the ganglia were washed with ASW for 10-15 min and then incubated with $5 \mu \mathrm{M}$ phorbol 12,13-diacetate (PDA; Sigma) for $30 \mathrm{~min}$ to induce maximal (and irreversible) PKC translocation to the membrane. After PDA treatment, the ganglia were washed for 5-15 min in ASW, and cell bodies were excised. The cells from four whole CNSs were pooled and homogenized in homogenization buffer (100 mm imidazole, $5 \mathrm{~mm}$ cysteine, $5 \mathrm{~mm} \mathrm{CaCl}_{2}, 20 \mathrm{~nm}$ acetyl pepstatin A, $1 \mu \mathrm{M}$ pepstatin A, $1 \mathrm{~mm}$ 4-(2-aminoethyl)-benzenesulfonyl fluoride, and $1 \mathrm{U} / \mathrm{ml}$ aprotinin, $\mathrm{pH}$ 7.5), and rat recombinant $\mathrm{m}$-calpain (Calbiochem) was added to reach a concentration of $6 \mathrm{U}$ per CNS. The reaction was stopped $0.5,1$, or $5 \mathrm{~min}$ later by the addition of sample buffer ( $8 \%$ glycerol, $5 \% \beta$-mercaptoethanol, $2.3 \%$ SDS, and $50 \mathrm{~mm}$ Tris, $\mathrm{pH}$ 6.8). Samples were boiled for $5 \mathrm{~min}$ and stored at $-70^{\circ} \mathrm{C}$ until use.

Proteins were resolved on $10 \%$ polyacrylamide gels and electrophoretically transferred to Hybond-P membranes (Amersham Biosciences, Piscataway, NJ). The blots were washed (three times for $5 \mathrm{~min}$ each) with Tris-buffered saline containing 0.1\% Tween 20 (TBST) (25 mu Tris-Cl, pH 7.5, $137 \mathrm{~mm} \mathrm{NaCl}, 2.7 \mathrm{~mm} \mathrm{KCl}$, and 0.1\% Tween 20), blocked for $1 \mathrm{hr}$ at room temperature with $5 \%$ nonfat dried milk in TBST, and then incubated with a 1:1000 dilution of primary antibodies (kindly provided by Dr. Wayne Sossin, McGill University, Montreal, Quebec, Canada) against the catalytic domain of the Aplysia Apl I and Apl II PKC isoforms overnight at $4^{\circ} \mathrm{C}$. After washing with TBST (three times for $5 \mathrm{~min}$ each), blots were incubated with an HRP-conjugated anti-rabbit secondary antibody (1:4000; Cell Signaling Technology, Beverly, MA), washed with TBST (three times for 5 min each), and the signal was detected with enhanced chemiluminescence (Amersham Biosciences).

Data analysis. In all experiments, duration of T-SW was measured by an observer who was blind to both training and drug treatment. The duration of T-SW was defined as the elapsed time from stimulus onset to the initial relaxation of the siphon from the contracted position. In all experiments, baseline duration of T-SW was determined by the average of three pretests. In one set of experiments (see Fig. 2), a fourth pretest was conducted in either normal ASW or with different regions of the CNS inactivated to ensure the efficacy of treatment. To keep the observer blind to this treatment, T-SW in this fourth pretest was measured by an independent observer. To examine ITM for sensitization, baseline T-SW and T-SW after training were analyzed with a single-factor ANOVA. After the ANOVA, individual time points were compared against baseline using Fisher's least significant difference test to determine significant sensitization.

\section{Results}

Site-specific intermediate-term memory is revealed by testing the site of tail shock

Since we identified previously an activity-dependent form of ITF at tail SN-MN synapses (Sutton and Carew, 2000), we first asked whether there might be a corresponding activity-dependent form of ITM in Aplysia. Thus, we examined whether site-specific ITM for sensitization of T-SW could be induced in a reduced behavioral preparation consisting of the surgically excised tail and mantle attached to the CNS. In this preparation (Fig. $1 A$ ), the ring ganglia (which contain tail SN-MN and tail SN-interneuron synapses) and abdominal ganglion (which contains the siphon MNs) are anatomically isolated and perfused independently. This experimental arrangement allows for rapid delivery and removal of drugs selectively to the ring ganglia, thus avoiding effects on motor components of siphon withdrawal. Before training, three 
pretest measurements of T-SW (elicited with a $0.5 \mathrm{sec}$ water jet) were taken at 15 min intervals to ensure a stable baseline response. Training consisted of a single 1.5 sec tail shock applied to: (1) the test site itself, (2) a different tail site ipsilateral to the test site, or (3) a site contralateral to the test site (Fig. 1A). We found that the single shock induced short-term memory (STM) for sensitization at the ipsilateral site (Fig. 1B) (15 min, $p<0.05$; 30-90 min, NS) and no sensitization at the contralateral site (15-90 min; all NS). The finding that sensitization was confined to the trained side is consistent with previous reports (Scholz and Byrne, 1987; Levenson et al., 2000) and likely reflects the lateralized nature of 5-HT release after tail shock (Marinesco and Carew, 2002). In contrast, testing at the shocked site revealed sensitization of T-SW that endured for $>90$ min after training (15-90 min; all $p<$ $0.05)$. These results confirm and extend those of Walters (1987), who first described site-specific enhancement of sensitization in Aplysia. Thus, our findings show that a single shock induces site-specific memory for sensitization that closely resembles ITM induced by repeated training trials, each of which extends well into the intermediateterm temporal domain (Fig. 1C).

To ensure that site-specific ITM requires plasticity within the CNS and is not merely a peripheral effect (e.g., resulting from shock-induced tissue damage, inflammation, etc.), we reversibly inactivated the CNS during training by perfusing the ring ganglia subchamber with modified ASW containing three times the normal concentration of $\mathrm{Mg}^{2+}$ and no added $\mathrm{Ca}^{2+}$ (Fig. 2, top). This manipulation abolishes T-SW by blocking synaptic activity within the ring ganglia as well as downstream activation of the abdominal ganglion, but the response completely recovers to baseline levels within 30 min of washout (Sutton et al., 2001). In normal ASW, as before, a single shock to the test site produced ITM for sensitization lasting >90 $\mathrm{min}$ (30-90 min; all $p<0.05$ ); this effect was completely blocked by CNS inactivation (Fig. 2, top) (30-90 min; all NS). Importantly, T-SW recovered completely to baseline levels in nontrained control preparations (30-90 min; all NS). These results demonstrate that site-specific ITM requires plasticity in the CNS and is not a peripherally mediated effect.

We next examined whether plasticity in the ring ganglia was sufficient for site-specific ITM by selectively blocking downstream activation of the abdominal ganglion during training through reversible conduction block of the pleural-abdominal nerves (Fig. 2, bottom). Blockade of the pleural-abdominal nerves completely abolished T-SW (Fig. 2, bottom, Pre4) as well as siphon withdrawal in response to a single shock to the test site (data not shown). However, when normal conduction of the pleural-abdominal connectives was restored, site-specific ITM was observed $30 \mathrm{~min}$ after training, and this sensitization persisted for $>90 \mathrm{~min}(30-90 \mathrm{~min}$; all $p<0.05)$. This site-specific ITM was not attributable to the inactivation procedure, be-
B.

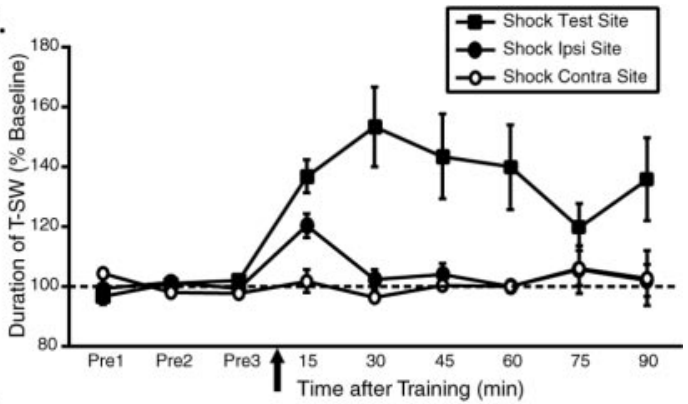

C.

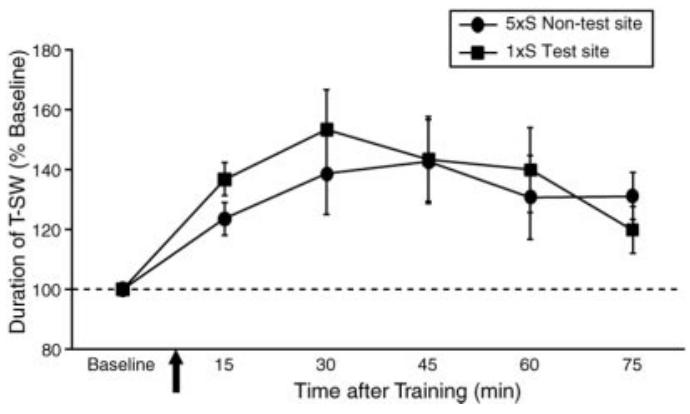

Figure 1. A single shock induces site-specific intermediate-term memory in a reduced preparation. $A$, Illustration of the reduced preparation depicting the sites on the tail used for training and testing. All pharmacological treatments were restricted to

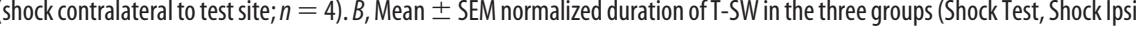

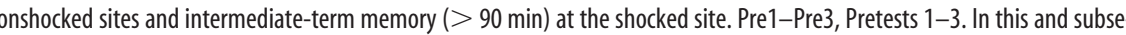
of intermediate-term memory for sensitization of T-SW in the reduced preparation induced either by repeated tail shocks to an data replotted from part B). With each pattern of experience, memory for sensitization persists for $>75 \mathrm{~min}$ after training and is of similar magnitude. Error bars represent SEM.

cause blockade of the pleural-abdominal connectives alone in nontrained preparations had no affect on baseline T-SW over the same time period (30-90 $\mathrm{min}$; all NS). These results indicate that activation of neural circuitry within the abdominal ganglion is not required for site-specific ITM. Thus, plasticity in the ring ganglia is both necessary and sufficient for the induction of sitespecific ITM.

\section{Site-specific ITM is mechanistically distinct from repeated-trial ITM}

We have shown previously that, in the ring ganglia, ITM produced by repeated, spaced tail shocks (Fig. 1C) requires protein synthesis for its induction and persistent PKA activation for its maintenance (Sutton et al., 2001). Because the plasticity required for site-specific ITM is also localized to the ring ganglia, we could now address whether site-specific ITM shares the same molecular mechanisms as repeated-trial ITM. We first examined whether the induction or expression of site-specific ITM requires translation by blocking protein synthesis in the ring ganglia with emetine $(100 \mu \mathrm{M})$ throughout training and testing (Fig. 3A). This concentration of emetine inhibits protein synthesis in isolated pleural-pedal ganglia by $>90 \%$ (data not shown) and blocks the induction of repeated-trial ITM (Sutton et al., 2001). In the presence or absence of emetine-containing ASW, a single shock to the test site produced significant ITM for sensitization lasting $>75$ min (15-75 min; all $p<0.05)$, demonstrating that site-specific ITM does not require new protein synthesis. 

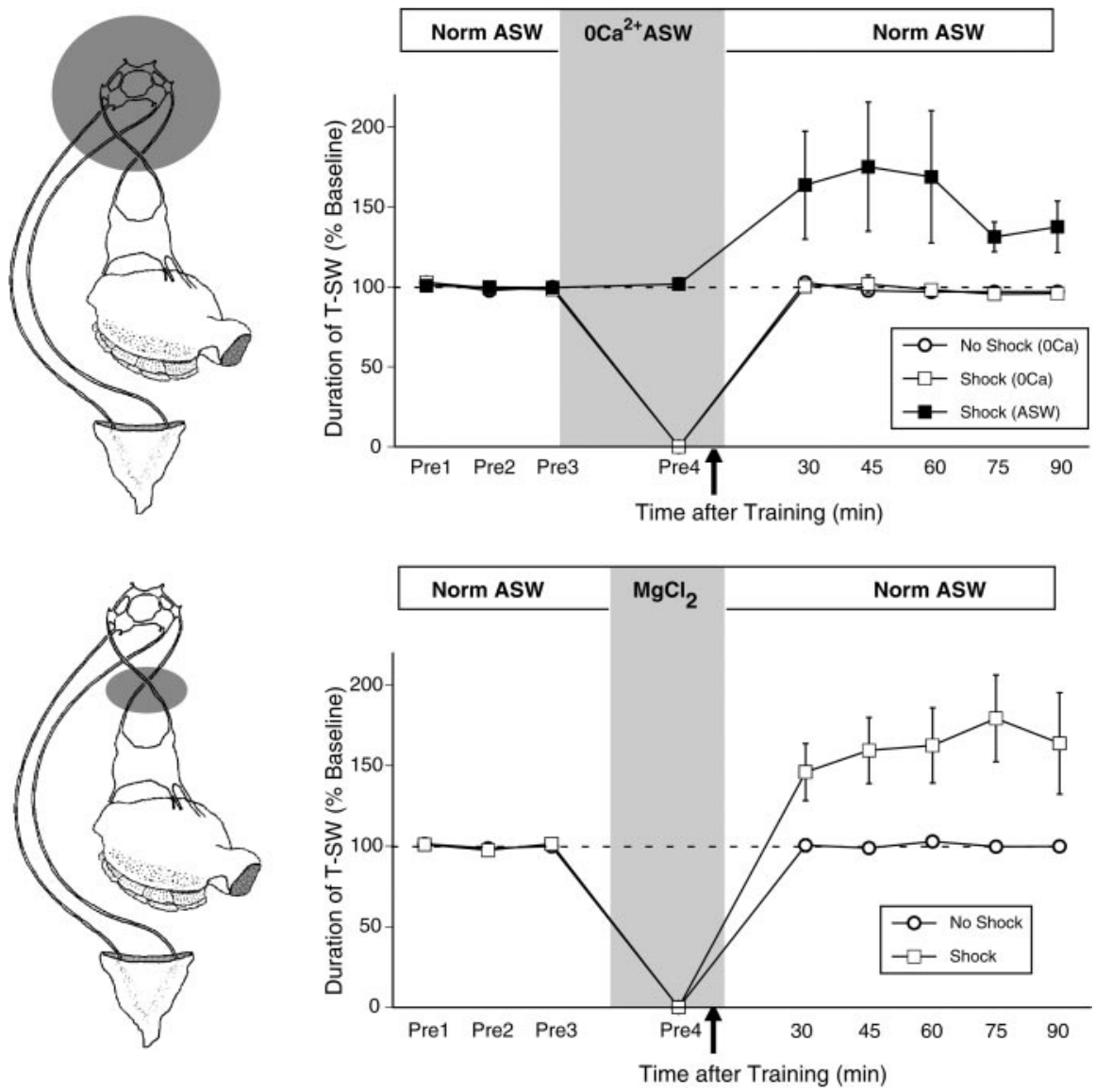

Figure 2. Plasticity in the ring ganglia is necessary and sufficient for site-specific ITM. Top, The CNS was inactivated by perfusion of the ring ganglia with modified ASW containing $3 \times \mathrm{Mg}^{2+}$ and no added $\mathrm{Ca}^{2+}$. The mean \pm SEM duration of T-SW in preparations that were trained with a single shock to the test site with $(n=4)$ or without $(n=6)$ CNS inactivation are shown. Recovery from CNS inactivation was assessed in nontrained preparations perfused with $3 \times \mathrm{Mg}^{2+}$ and no Ca ${ }^{2+}$ ASW in parallel $(n=3)$. Control preparations treated with normal ASW (Norm ASW) demonstrated robust site-specific ITM for sensitization at the shocked site; this effect was completely blocked by CNS inactivation. After washout, T-SW recovered completely to baseline levels in nontrained preparations. Bottom, Inactivation of the abdominal ganglion during training was achieved by isolating short segments $\left(\sim 1-2 \mathrm{~mm}\right.$ ) of both pleural-abdominal nerves in a separate subchamber and substituting ASW with isotonic $\mathrm{MgCl}_{2}$ to block action potential conduction. After a complete bath exchange of the subchamber, a fourth pretest of T-SW by an independent observer was taken to verify functional blockade. After this test, the $\mathrm{MgCl}_{2}$ remained in the subchamber for 5 min before the application of a single tail shock to the test site and for an additional 5 min period after training, after which perfusion of the subchamber with ASW resumed. The mean \pm SEM duration of T-SW in preparations that received either a single shock to the test site $(n=5)$ or no shock $(n=3)$ with the abdominal ganglion inactivated are shown. Inactivation of the abdominal ganglion had no effect on the induction of site-specific ITM. Pre1-Pre4, Pretests 1-4.

We next examined the effect of inhibiting PKA activity on the induction and maintenance of site-specific ITM. As shown in Figure 3B, application of the PKA inhibitor KT5720 $(10 \mu \mathrm{M})$ for 30 min before and during training had no effect on the induction of site-specific ITM (15-90 min; all $p<0.05$ for both groups). To examine whether PKA activity may be required for the maintenance of site-specific ITM, KT5720 was applied $30 \mathrm{~min}$ after training (Fig. 3C). Again, KT5720 had no effect on site-specific ITM (15-90 min; all $p<0.05$ for both groups). We should emphasize that this concentration of KT5720 and exact experimental procedure reversibly abolish the expression of repeated-trial ITM (Sutton et al., 2001). Thus, unlike repeated-trial ITM, site-specific ITM does not require protein synthesis or PKA activity for induction or maintenance. These results indicate that two phenotypically similar but mechanistically distinct forms of memory for sensitization can be observed in the intermediate-term temporal domain.
Maintenance of site-specific ITM involves persistent activation of $\mathrm{PKC}$ Although the maintenance of site-specific ITM does not require PKA activity, another candidate signaling molecule that could contribute to the maintenance of site-specific ITM is PKC, which also becomes persistently activated in tail SNs in response to patterns of 5-HT exposure that induce ITF and LTF (Sossin et al., 1994; Sossin, 1997; Zhang et al., 1997; Yanow et al., 1998). Moreover, similar to the behavioral results described above, activity-dependent ITF at tail SN-MN synapses can be distinguished from ITF induced by repeated 5-HT pulses in that it does not require protein synthesis for induction, and its maintenance requires persistent activation of PKC but not PKA (Sutton and Carew, 2000).

To define the role of PKC in sitespecific ITM requires examining its contribution to both the induction and maintenance of this form of memory. We first turned our attention to the question of maintenance (we address the specific role of PKC in the induction of site-specific ITM in experiments described below). To explore whether a persistent activation of PKC might contribute to the maintenance of site-specific ITM, we applied the reversible PKC inhibitors chelerythrine $(50 \mu \mathrm{M})$ and bisindolylmaleimide I $(10 \mu \mathrm{M})$ to the ring ganglia after site-specific ITM was induced (Fig. 4). Importantly, each of these inhibitors targets the catalytic domain of PKC (Herbert et al., 1990; Toullec et al., 1991) and therefore inhibits PKCmediated effects downstream of its activation. As shown in Figure 4A, a single shock to the test site induced comparable levels of sensitization at 15 and $30 \mathrm{~min}$ after training in both shocked groups (15-30 min; both $p<0.05$ in both groups). However, whereas ITM was preserved in vehicle-treated preparations (45-75 min; all $p<0.05)$, ITM was abolished in those preparations treated with chelerythrine (45-75 min; all NS). When chelerythrine was washed out of the ring ganglia subchamber, significant sensitization was again observed, and this recovery of ITM was comparable with the level of sensitization observed in vehicle-treated preparations at the same time points (90-120 min; $p<0.05$ in both groups). Importantly, chelerythrine had no effect on baseline T-SW in nontrained preparations (NS) treated over the same time period (15-120 min; all NS). We observed similar results with the PKC inhibitor bisindolylmaleimide (Fig. 4B) Again, site-specific ITM was very similar in both shocked groups before drug treatment (30 min test) (Fig. 4B). Although site-specific ITM was maintained in the presence of the vehicle $(0.5 \%$ DMSO; $45-75 \mathrm{~min}$; all $p<0.05)$, it was completely blocked by bisindolylmaleimide ( $45-75 \mathrm{~min}$; all NS). As with chelerythrine, the block of ITM was reversible: significant sensitization comparable with that expressed in 
A.

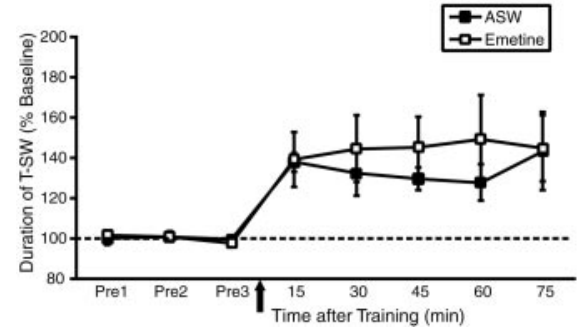

B.

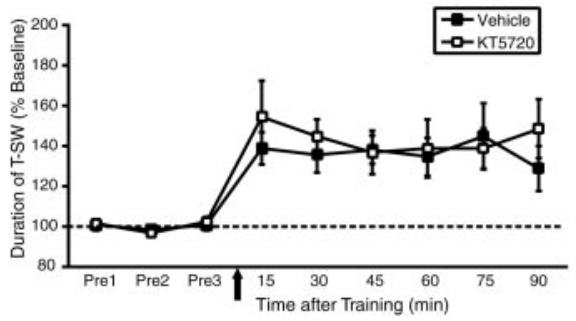

C.

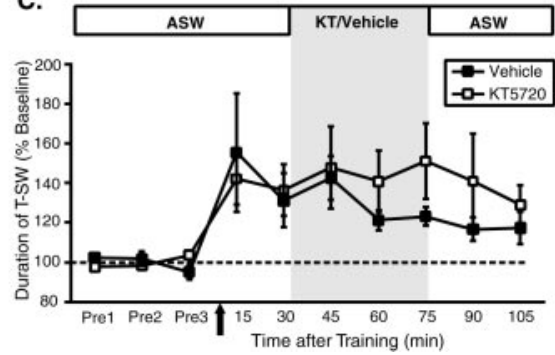

Figure 3. Site-specific ITM has a unique molecular mechanism. A, Mean \pm SEM normalized duration of T-SW over time in preparations treated with or without the protein synthesis inhibitor emetine (100 $\mu \mathrm{M})$ throughout the experiment ( $n=6$ per group). $B$, Mean \pm SEM normalized duration of T-SW over time in preparations treated with the PKA inhibitor KT5720 $(10 \mu \mathrm{M})$ orvehicle $(1 \% \mathrm{DMSO})$ for 30 min before and during training with a single shock to the test site ( $n=5$ per group). KT5720 -vehicle was added to the ring ganglia subchamber after completion of the pretests and was washed out immediately after training. C, Mean \pm SEM normalized duration of T-SW over time in preparations treated with $10 \mu \mathrm{M} \mathrm{KT5720}(n=6)$ or vehicle $(n=7)$ beginning 30 min after training with a single shock to the test site. KT5720-vehicle (KT/Vehicle) was washed out immediately after the 75 min post-test. Drug-vehicle application is indicated by shading. Pre1-Pre3, Pretests 1-3.

vehicle-treated preparations was observed after bisindolylmaleimide washout (105-120 min; both $p<0.05$ ). Finally, bisindolylmaleimide applied to nontrained preparations did not affect baseline T-SW (15-120 min; all NS). These results indi-

A.

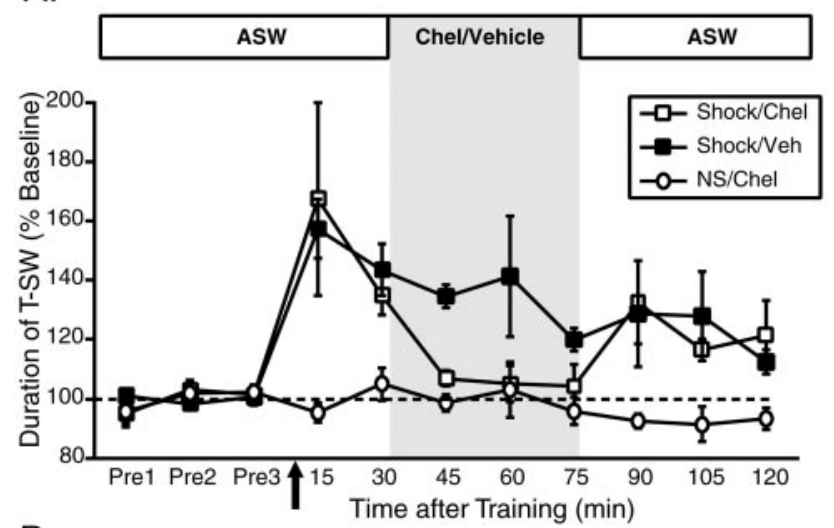

B.

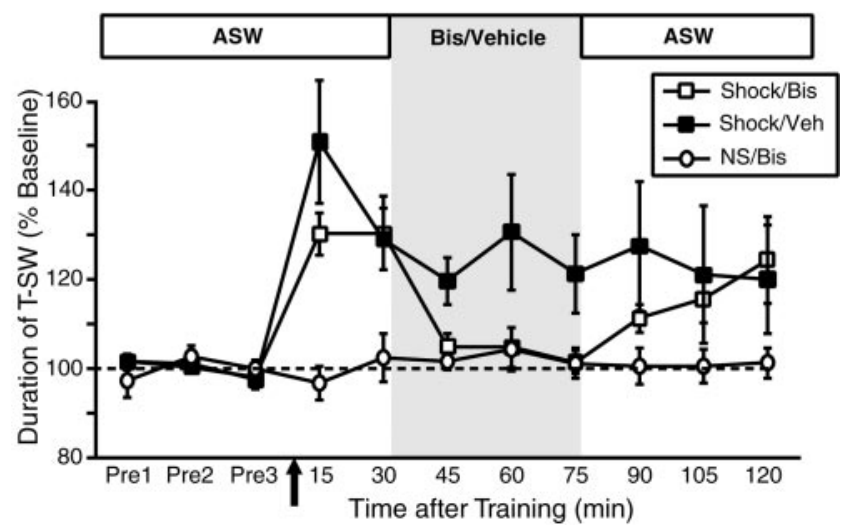

Figure 4. Site-specific ITM is maintained by a persistent activation of PKC. A, Mean \pm SEM normalized duration of T-SW over time in preparations treated with the PKC inhibitor chelerythrine (Chel) $(50 \mu \mathrm{m} ; n=6)$ or vehicle (Veh) (distilled water; $n=7)$ beginning 30 min after training with a single shock to the test site. Nontrained preparations treated with chelerythrine over the same time period $(n=3)$ were examined in parallel. $B$, Mean \pm SEM normalized duration of T-SW over time in preparations treated with the PKC inhibitor bisindolylmaleimide I (Bis) $(10 \mu \mathrm{M} ; n=6)$ or vehicle $(0.5 \%$ DMSO; $n=6)$ beginning 30 min after training with a single shock to the test site. Nontrained preparations treated with bisindolylmaleimide I over the same time period ( $n=3$ ) were examined in parallel. In both experiments, drug-vehicle was washed out immediately after the $75 \mathrm{~min}$ post-test. Drug-vehicle application is indicated by shading. Pre1-Pre3, Pretests $1-3$. cate that site-specific ITM is maintained, at least in part, by a persistent activation of $\mathrm{PKC}$, a characteristic that bears strong resemblance to the features of activity-dependent ITF at tail SN-MN synapses (Sutton and Carew, 2000).

\section{Persistent PKC activation in the maintenance of site-specific} ITM involves a PKM

The data described above suggest that site-specific ITM is maintained by a persistent activation of PKC that is generated rapidly (i.e., by a single $1.5 \mathrm{sec}$ tail shock) in a protein synthesisindependent manner. These features of site-specific ITM are suggestive of possible mechanisms that could generate the persistent $\mathrm{PKC}$ activation required for memory maintenance. One possible mechanism involves limited proteolysis of the PKC enzyme itself, generating a PKM (Inoue et al., 1977; Suzuki et al., 1992; Sacktor et al., 1993).

PKC exists as a single polypeptide, with an N-terminal regulatory domain and a C-terminal catalytic domain. Under basal conditions, $\mathrm{PKC}$ resides in the cytosol, and the regulatory domain acts as a pseudosubstrate for the catalytic domain, thus providing autoinhibition of PKC-dependent phosphorylation. In response to modulatory inputs (e.g., 5-HT) acting through phospholipase C-coupled signaling pathways, $\mathrm{PKC}$ can translocate to the membrane, inducing a conformational change in the protein that relieves the autoinhibition by the regulatory domain, thus producing an active form of the enzyme that can phosphorylate substrate proteins. However, this mode of PKC activation will be transient, lasting only as long as the acute elevation of intracellular second messengers persists. One way to convert this normally transient $\mathrm{PKC}$ activation into a persistent activation is through neuronal activity coincident with the modulatory inputs. For example, activity can lead to large increases in $\mathrm{Ca}^{2+}$ influx, which in turn can activate the $\mathrm{Ca}^{2+}$-dependent protease calpain. When active, calpain cleaves activated PKC between the regulatory and catalytic domains, inducing a physical separation of these two regions of the enzyme (Kishimoto et al., 1983; Suzuki et al., 1992). This process liberates a PKM that will be persistently active, because it is no longer associated with the autoinhibitory regulatory domain (Inoue et al., 1977; Sessoms et al., 1992; Sacktor et al., 1993).

How might this proteolytic generation of PKM contribute to site-specific ITM? First, the subpopulation of tail SNs that innervate the shocked site should experience the modulatory effects of 5-HT coincident with their own activation during tail shock. Second, subsequent test stimuli delivered to that site should recruit that same population of SNs. Therefore, in these SNs, the interaction of their activation with the modulatory effects of tail shock 
may lead to activation of calpain (driven by activity) coincident with $\mathrm{PKC}$ translocation (driven by 5-HT), resulting in proteolytic generation of a PKM. Importantly, the proteolytic induction mechanism in those activated SNs would be rapid and independent of protein synthesis, which are features shared by sitespecific ITM. We explored whether the persistent activation of PKC underlying the maintenance of site-specific ITM involves a PKM in three ways. First, we examined the effects of another PKC inhibitor, calphostin C, which interacts specifically with the regulatory domain of PKC. Second, we examined whether endogenous Aplysia PKC was a substrate for calpain. Finally, we examined the effect of blocking calpain activity on the induction and maintenance of site-specific ITM.

\section{Calphostin $\mathrm{C}$ blocks the induction but not the maintenance of} site-specific ITM

Because calphostin $\mathrm{C}$ interacts with the regulatory domain of PKC (Kobayashi et al., 1989) and effectively inhibits persistent PKC activity in Aplysia that is independent of PKM (Sossin, 1997), the hypothesized role of a proteolytically generated PKM generates clear predictions regarding the effects of calphostin $\mathrm{C}$ on the induction and maintenance of site-specific ITM. First, calphostin $\mathrm{C}$ should block the induction of site-specific ITM when applied before training, because PKC translocation (via the regulatory domain) to the membrane is thought to be required for calpain-dependent cleavage (Kishimoto et al., 1983). However, once induced, the maintenance of site-specific ITM should be resistant to inhibition by calphostin $\mathrm{C}$, because the regulatory domain would no longer be associated with a PKM. To examine these predictions, we first applied calphostin C $(5 \mu \mathrm{M})$ or its vehicle (1\% DMSO) to the ring ganglia subchamber for $45 \mathrm{~min}$ before training with a single shock to the test site and then washed out the drug immediately after training. As shown in Figure $5 \mathrm{~A}$, calphostin $\mathrm{C}$ completely blocked the induction of site-specific ITM (15-90 min; all NS). However, when calphostin C was applied $30 \mathrm{~min}$ after induction for the same $45 \mathrm{~min}$ incubation period (Fig. 5B), it had no effect on the maintenance of sitespecific ITM (45-75 min; $p<0.05$ for both calphostin C-treated and vehicle-treated groups). Finally, calphostin $\mathrm{C}$ did not inhibit baseline T-SW assessed in nontrained control preparations examined in parallel. These results indicate that PKC plays a direct role not only in the maintenance of site-specific ITM (Fig. 4) but in its induction as well. Moreover, the observation that calphostin $\mathrm{C}$ blocks the induction but not the maintenance of sitespecific ITM is consistent with the hypothesis that the persistent $\mathrm{PKC}$ activation required for maintenance derives from a PKM.

\section{Aplysia PKC isoforms are proteolyzed by calpain}

We next asked whether endogenous Aplysia PKC is a substrate for calpain. To address this issue, isolated CNS homogenates were made from buccal, pleural-pedal, cerebral, and abdominal ganglia treated previously with phorbol ester ( $5 \mu \mathrm{M}$ PDA) for $30 \mathrm{~min}$ to induce membrane translocation of PKC. Equal quantities of the homogenate were then incubated with or without rat recombinant calpain (6 U per CNS) for the times indicated in Figure 6. The samples were separated on $10 \%$ polyacrylamide gels, blotted, and probed with antibodies raised against the catalytic domains of the $\mathrm{Ca}^{2+}$-dependent (classical) PKC isoform Apl I and the $\mathrm{Ca}^{2+}$-independent (novel) PKC isoform Apl II. As shown in Figure $6 A$, the Apl I holoenzyme $(\sim 70 \mathrm{kDa})$ is rapidly (within 30 $\mathrm{sec}$ ) proteolyzed by calpain, yielding a major catalytic fragment of $\sim 30 \mathrm{kDa}$. Calpain also proteolyzed the Apl II holoenzyme ( $\sim 90$ $\mathrm{kDa}$ ), albeit less rapidly than it did Apl I, yielding a major catalytic
A.

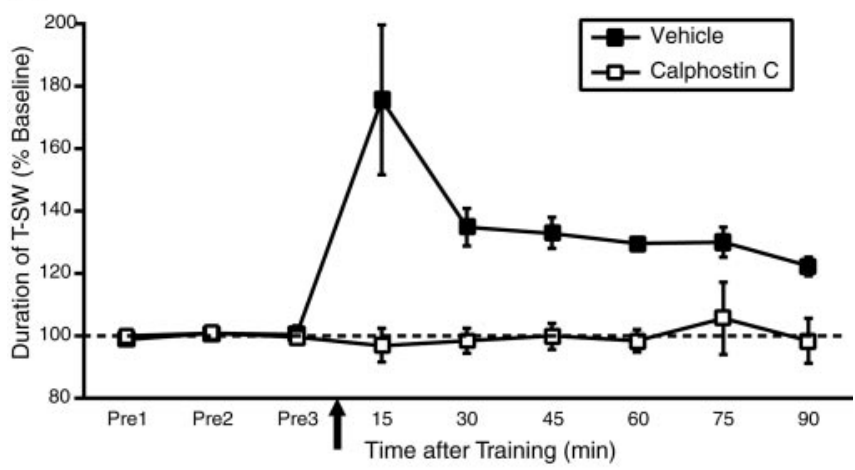

B.
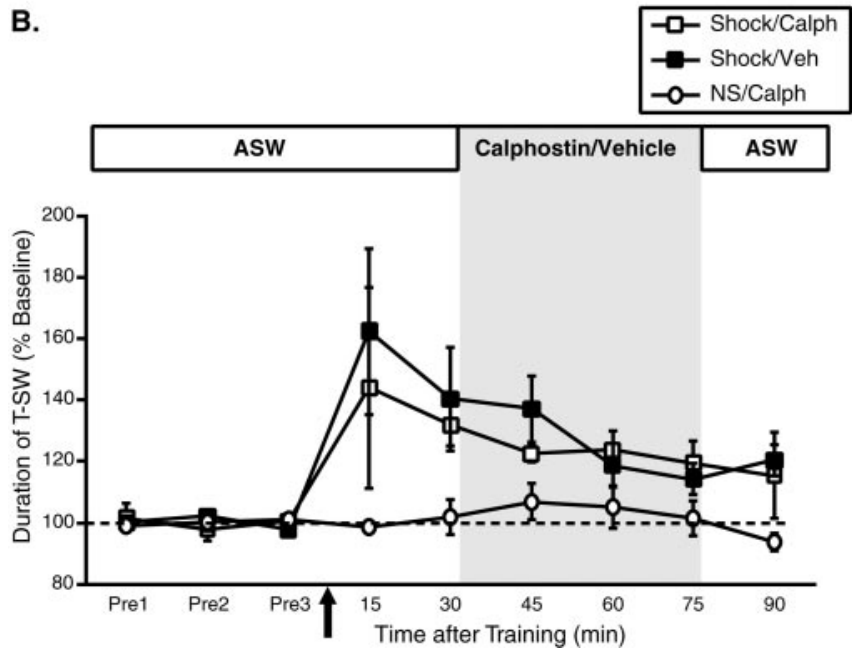

Figure 5. Calphostin C, a PKC inhibitor interacting with the regulatory domain, blocks the induction but not the maintenance of site-specific ITM. $A$, Mean \pm SEM normalized duration of T-SW over time in preparations treated with the PKC inhibitor calphostin C $(5 \mu \mathrm{M})$ or vehicle $(1 \%$ DMSO) for 45 min before and during training with a single shock to the test site $(n=6$ per group). Calphostin-vehicle was added to the ring ganglia subchamber after completion of the pretests and was washed out immediately after training. Calphostin C completely blocked the induction of site-specific ITM. B, Mean \pm SEM normalized duration of T-SW over time in preparations treated with $5 \mu \mathrm{m}$ calphostin C (Calph; $n=6)$ or vehicle (Veh; $n=8)$ beginning $30 \mathrm{~min}$ after training with a single shock to the test site. Nontrained preparations treated with calphostin C over the same time period $(n=3)$ were examined in parallel. Calphostin-vehicle was washed out immediately after the 75 min post-test. Drug-vehicle application is indicated by shading. The maintenance of site-specific ITM was not affected by calphostin C. Note that the calphostin C incubation time in this experiment is identical to that in $A$. Pre1-Pre3, Pretests 1-3.

fragment of $\sim 45 \mathrm{kDa}$ (Fig. $6 \mathrm{~B}$ ). These results demonstrate that the two major endogenous PKC isoforms in Aplysia are substrates for calpain.

Because recombinant calpain can generate PKM in cell extracts, it was logical to examine whether a PKM could be generated in situ by pairing 5-HT or phorbol ester with stimulation conditions that enhance calcium influx. To this end, we treated isolated pleural-pedal ganglia with 5 -HT $(50 \mu \mathrm{M}, 15 \mathrm{~min})$ or 5 $\mu \mathrm{M}$ PDA (to activate PKC) paired either with high-K ${ }^{+}$ASW or with the calcium ionophores A23187 (5-40 $\mu \mathrm{M})$ and ionomycin $(100 \mu \mathrm{M})$. However, the results gained from a number of experiments failed to reveal a particular combination of treatments whereby a catalytic fragment of Apl I or Apl II could be reliably detected by Western blotting under our conditions. Several other studies have likewise reported that endogenous generation of PKM is very difficult to detect (Shea et al., 1994), and a failure to observe PKM by immunoblotting is not uncommon (Grünbaum 
and Müller, 1998). In those cases in which proteolytic generation of PKM can be reliably detected (Powell et al., 1994), it represents an extremely small fraction of the total PKC pool. Thus, it is likely that our immunodetection methods simply lack the sensitivity required to detect low levels of endogenous PKM.

\section{Calpain inhibitors block the induction} but not the maintenance of site-specific ITM

Given that endogenous Aplysia PKC is a substrate for calpain, we next examined the effect of blocking calpain activity on the induction and maintenance of sitespecific ITM. In these studies, we used two cell-permeable but structurally distinct calpain inhibitors: $N$-acetyl-leucyl-leucylmethional (ALLM) $(100 \mu \mathrm{M})$ and MDL28170 $(100 \mu \mathrm{M})$. We first examined their effect on the induction of ITM by applying them (or vehicle, $1 \%$ DMSO) to the ring ganglia subchamber for 45 min before training with a single shock to the test site and then washing them out immediately after training (Fig. 7A). In vehicle-treated preparations, as before, a single shock to the test site produced robust ITM for sensitization lasting $>90 \mathrm{~min}$ (15-90 $\mathrm{min}$; all $p<0.05)$. In contrast, ALLM completely blocked the induction of site-specific ITM (15 min, $p<0.05 ; 30-90 \mathrm{~min}$, all NS); similar effects were also observed with MDL28170 (15-90 min; all NS). Neither ALLM nor MDL28170 pretreatment affected baseline T-SW assessed in nontrained control preparations examined in parallel. These results indicate that calpain activation is required for the induction of site-specific ITM. In contrast to their effect on induction, neither ALLM nor MDL28170 had any effect on the maintenance of site-specific ITM once it had been induced normally (Fig. 7B) (15-120 $\mathrm{min} ; p<0.05$ for all groups). These results further support the hypothesis that a PKM is generated during behavioral training, because calpain activity is required only during the time period when PKC would be accessible for cleavage.

\section{Discussion}

Our results suggest two main conclusions: First, site-specific ITM uses molecular mechanisms that are distinct from those used by repeated-trial ITM. Second, site-specific ITM is maintained by a persistent activation of $\mathrm{PKC}$, which is achieved through calpaindependent generation of a PKM.

\section{Mechanistically distinct forms of ITM for sensitization in Aplysia}

Different patterns of behavioral training and testing reveal two forms of ITM for sensitization with unique molecular mechanisms. When training and testing are each directed at separate ipsilateral sites on the tail, a single shock induces STM (lasting $<30 \mathrm{~min}$ ) that is independent of protein synthesis, whereas repeated spaced shocks induce ITM (lasting $>120 \mathrm{~min}$ ) that requires protein but not RNA synthesis as well as long-term memory (LTM) (>24 hr) that requires both (Castellucci et al., 1989; Levenson et al., 2000; Sutton et al., 2001). The maintenance of repeated-trial ITM requires a persistent activation of PKA in the ring ganglia (Sutton et al., 2001). Here, we found that a single shock can also induce ITM for sensitization at the site where
B.

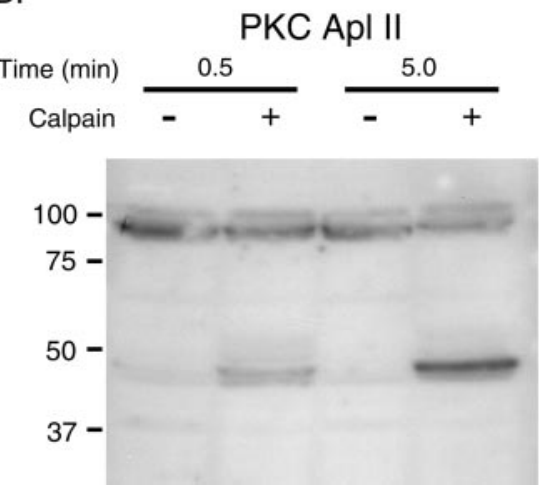




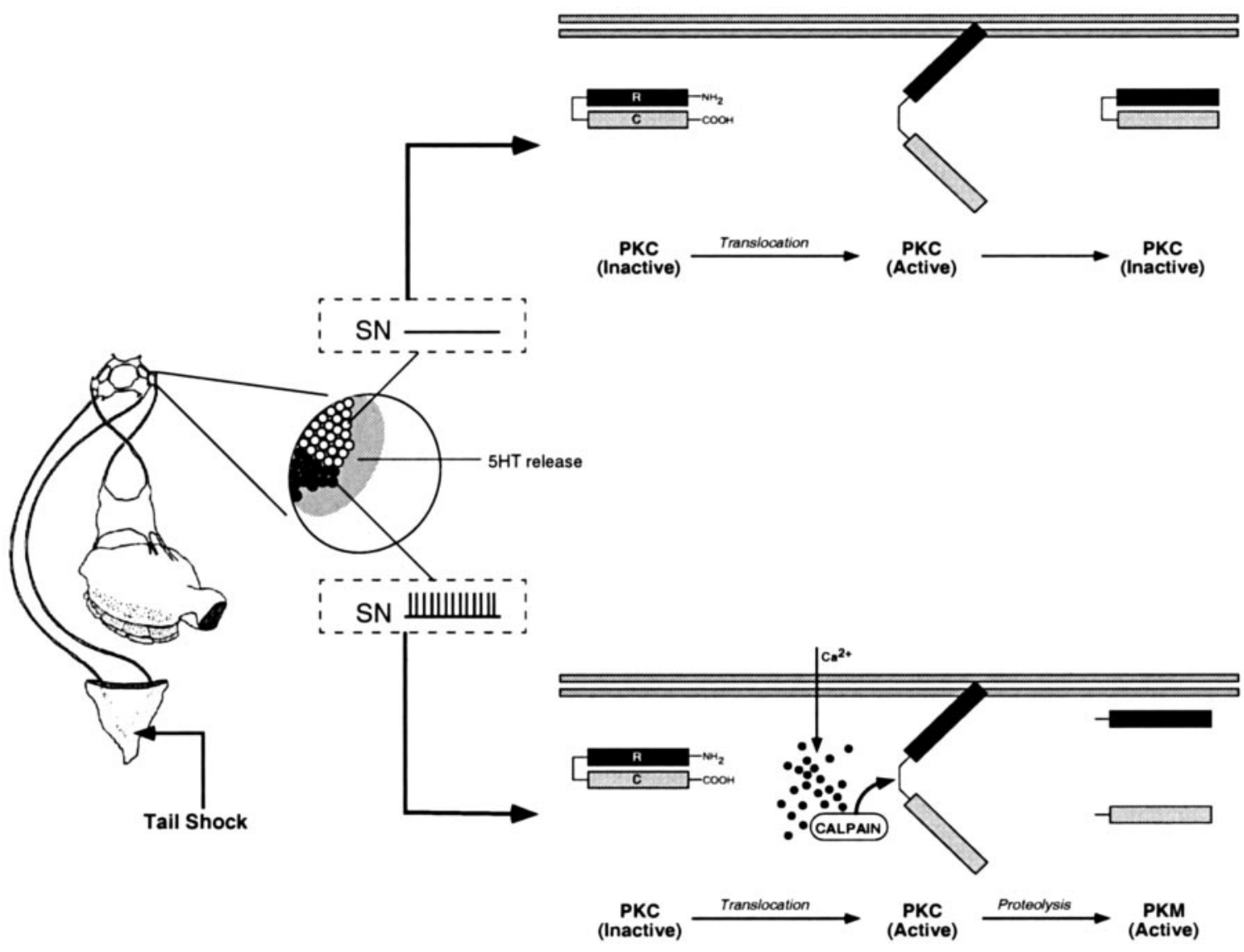

Figure 8. A model for the induction and maintenance of site-specific ITM. A schematic representation of the proposed molecular events related to PKC activation in tail SNs (or possibly interneurons activated by these SNs) after tail shock is shown. Under basal conditions, PKC is retained in the cytosol, and its catalytic activity is inhibited by the N-terminal regulatory domain (R) that acts as a pseudosubstrate for the (-terminal catalytic domain (C). A single tail shock, regardless of location, will elicit 5 -HT release (shaded area) that is distributed across the entire population of SNs and their synapses (Marinesco and Carew, 2002). 5-HT, acting through phospholipase C-coupled receptors (Li et al., 1995), stimulates translocation of PKC to the membrane, inducing a conformational change that relieves the autoinhibition of PKC activity by the regulatory domain. In SNs with receptive fields that lie outside the region of the tail receiving shock (open circles and top), 5-HT-induced PKC activation will be transient, enduring only as long as the short-term modulatory effects of 5-HT on second messengers persist. Conversely, the SNs innervating the shocked site will be activated (Walters et al., 1983a,b) coincident with 5-HT exposure (filled circles and bottom). SN activation increases intracellular $\mathrm{Ca}^{2+}$ influx, stimulating the $\mathrm{Ca}^{2+}$-activated protease calpain. Calpain, in turn, proteolyses activated PKC, generating persistently active PKMs. Thus, the induction of site-specific sensitization entails the proteolytic processing of activated PKC, the product of which (PKM) serves to maintain the memory in the intermediate-term temporal domain.

training is administered. Similar to repeated-trial ITM, this sitespecific form of ITM is mediated by plasticity within the ring ganglia. However, site-specific ITM does not require new protein synthesis for induction or PKA for maintenance; it is maintained, at least in part, through a persistent activation of PKC. Thus, the molecular mechanisms underlying these two forms of ITM are mechanistically distinct, illustrating the point that in addition to unique molecular requirements that distinguish between phases of memory in Aplysia and many other systems, multiple forms of memory with distinct molecular requirements exist within a given temporal phase. This feature of ITM adds to an emerging theme in recent studies of memory and synaptic plasticity in a range of systems that the same functional endpoint can be achieved through multiple molecular mechanisms whether that endpoint is long-term potentiation (LTP) (Kang et al., 1997; Patterson et al., 2001) or long-term depression (Oliet et al., 1997; Huber et al., 2000) at CA3-CA1 synapses in the hippocampus, ITF or LTF of SN-MN synapses in Aplysia (Casadio et al., 1999; Bailey et al., 2000; Sutton and Carew, 2000; Sherff and Carew, 2002), or LTM for conditioned taste aversion (Berman and Dudai, 2001).

The ability to uncouple induction and maintenance mecha- nisms for memory in Aplysia allowed us to answer the question posed at the outset of this study: Our results show conclusively that, at least in the case of ITM, the unique molecular mechanisms involved in the induction of site-specific and repeated-trial ITM do not converge on a common molecular mechanism for maintaining the memory. Rather, each form of ITM is maintained by a distinct mechanism (persistent activation of PKC and PKA, respectively), demonstrating that the maintenance of memory over the same temporal domain can be achieved through multiple molecular strategies.

\section{PKM in synaptic plasticity and memory}

We found that inhibitors that target the catalytic domain of PKC reversibly blocked the expression of site-specific ITM when administered 30 min after training. Moreover, they blocked ITM downstream of mechanisms underlying maintenance, because in each case, ITM recovered to normal control levels when PKC activity was restored. These results suggest that site-specific sensitization is maintained, at least in part, through a persistent activation of PKC. In contrast, calphostin $\mathrm{C}$, a PKC inhibitor that interacts specifically with the regulatory domain of PKC, was ineffective in blocking expression when applied after training. 
However, it did block the induction of site-specific ITM when applied before training and has been shown previously to block persistent PKC activity in Aplysia that is independent of PKM (Sossin, 1997). Thus, the differential effects of calphostin C on the induction and expression of site-specific ITM raise the possibility that the persistent PKC activation required for the maintenance of site-specific ITM is achieved through the proteolytic generation of a PKM that is no longer physically associated with the regulatory domain. This hypothesis is further supported by the observations that: (1) calpain, a $\mathrm{Ca}^{2+}$-activated protease that is known to induce PKM formation in several systems (Inoue et al., 1977; Kishimoto et al., 1983; Sessoms et al., 1992; Sato et al., 1997), proteolyses endogenous Aplysia PKC, and (2) inhibition of calpain activity specifically blocked the induction but not the maintenance of site-specific ITM. These results suggest that the persistent activation of $\mathrm{PKC}$ required for the maintenance of sitespecific ITM derives from a PKM.

A working model to explain the induction and maintenance of site-specific ITM is presented in Figure 8. Tail shock induces 5-HT release (Marinesco and Carew, 2002) as well as activation of a subpopulation of tail SNs that innervate the shocked site (Walters et al., 1983a,b; Walters, 1987). 5-HT, acting through phospholipase C-coupled receptors ( $\mathrm{Li}$ et al., 1995), stimulates the translocation and transient activation of PKC in tail SNs (Sacktor and Schwartz, 1990; Sossin and Schwartz, 1992). Studies of the serotonergic innervation in the ring ganglia, coupled with direct amperometric measurements of 5-HT release, suggest that 5 -HT release is distributed across the majority of the, if not the entire, population of SN synapses (Zhang et al., 1991; Marinesco and Carew, 2002). Thus, PKC will be activated in all tail SNs exposed to 5-HT as a consequence of tail shock. However, in those SNs with receptive fields that lie outside the shocked area, 5-HT-induced PKC activation is transient, lasting only as long as the acute effects of 5-HT are exerted in these SNs. Therefore, when testing a site different from the shocked site itself, only STM is observed, because this test recruits a population of SNs that have experienced only short-term modulatory effects of 5-HT. In contrast, testing the site of shock recruits a subpopulation of "on-field" SNs that are exposed to 5-HT in conjunction with their own activation. The activation of on-field SNs by shock increases $\mathrm{Ca}^{2+}$ influx (Eliot et al., 1993) and activates calpain, which proteolytically converts the transiently active PKC into a persistently active PKM. Thus, the induction of site-specific sensitization entails the proteolytic processing of PKC, and in turn, the catalytic fragments generated by such processing are, at least in part, responsible for maintaining the memory in the IT range. We should emphasize that although the model is based on observed plasticity in tail SNs, it could also apply to interneuronal sites of the T-SW circuit that are located in the ring ganglia (Cleary and Byrne, 1993).

A critical role for PKM in memory and synaptic plasticity has also been suggested by work in other systems. For example, calpain is activated in the dendrites of CA1 hippocampal neurons during LTP induction (Vanderklish et al., 1995), and inhibition of calpain activity blocks LTP in the CA1 region (del Cerro et al., 1990; Vanderklish et al., 1996). One important consequence of calpain activation for LTP in the hippocampus is the induction of persistently active PKC through proteolytic generation of PKM (Sessoms et al., 1992; Suzuki et al., 1992; Sacktor et al., 1993; Powell et al., 1994). Finally, other studies suggest an important role for PKM deriving from the PKC $\zeta$ isoform in the protein synthesis-dependent late phase of LTP (L-LTP) in area CA1 (Sacktor et al., 1993; Osten et al., 1996), which appears to be both necessary and sufficient for maintaining L-LTP (Ling et al., 2002). These results suggest that in CA1 of the hippocampus, a protein synthesis-dependent mechanism for PKM formation may be recruited in the maintenance of late-phase LTP.

Two other studies have also demonstrated an important role for PKM in memory processing. First, Grünbaum and Müller (1998) found that multiple-trial olfactory conditioning in the honeybee induces two distinct phases of persistent PKC activation in the antennal lobes: an early phase requiring proteolysis but not protein synthesis and a late phase that required protein synthesis. Interestingly, blockade of the early proteolysisdependent phase disrupted an intermediate phase of memory for olfactory conditioning. Similarly, recent work by Drier et al. (2002) has demonstrated that post-training induction of a PKM transgene enhances the formation of long-lasting memory for olfactory conditioning in Drosophila. The induction of PKM enhances memory after massed but not spaced training and partially rescues the memory deficits of radish mutants. These latter effects suggest a role for PKM in both an intermediate phase of memory ("anesthesia-resistant memory") and long-term memory for olfactory conditioning in Drosophila (Tully et al., 1994). Together, these two studies suggest that persistent activation of $\mathrm{PKC}$, generated either via proteolysis or via new macromolecular synthesis, contributes to intermediate-term and long-term memories, respectively. Thus, although a role for PKC activation in cellular and behavioral plasticity has been well established, converging lines of evidence from several systems now implicate a novel role for PKM in maintaining enduring forms of both synaptic plasticity and memory.

\section{References}

Bailey CH, Giustetto M, Zhu H, Chen M, Kandel ER (2000) A novel function for serotonin-mediated short-term facilitation in Aplysia: conversion of a transient, cell-wide homosynaptic hebbian plasticity into a persistent, protein synthesis-independent synapse-specific enhancement. Proc Natl Acad Sci USA 97:11581-11586.

Bao J-X, Kandel ER, Hawkins RD (1998) Involvement of presynaptic and postsynaptic mechanisms in a cellular analog of classical conditioning at Aplysia sensory-motor neuron synapses in isolated cell culture. J Neurosci 18:458-466.

Berman DE, Dudai Y (2001) Memory extinction, learning anew, and learning the new: dissociations in the molecular machinery of learning in cortex. Science 291:2417-2419.

Casadio A, Martin KC, Giustetto M, Zhu H, Chen M, Bartsch D, Bailey CH, Kandel ER (1999) A transient, neuron-wide form of CREB-mediated long-term facilitation can be stabilized at specific synapses by local protein synthesis. Cell 99:221-237.

Castellucci VF, Blumenfeld H, Goelet P, Kandel ER (1989) Inhibitor of protein synthesis blocks long-term behavioral sensitization in the isolated gill-withdrawal reflex of Aplysia. J Neurobiol 20:1-9.

Cleary LJ, Byrne JH (1993) Identification and characterization of a multifunction interneuron contributing to defensive arousal in Aplysia. J Neurophysiol 70:1767-1776.

del Cerro S, Larson J, Oliver MW, Lynch G (1990) Development of hippocampal long-term potentiation is reduced by recently introduced calpain inhibitors. Brain Res 530:91-95.

Drier EA, Tello MK, Cowan M, Wu P, Blace N, Sacktor TC, Yin JCP (2002) Memory enhancement and formation by atypical PKM activity in Drosophila melanogaster. Nat Neurosci 5:316-324.

Eliot LS, Kandel ER, Siegelbaum SA, Blumenfeld H (1993) Imaging terminals of Aplysia sensory neurons demonstrates role of enhanced $\mathrm{Ca}^{2+}$ influx in presynaptic facilitation. Nature 361:634-637.

Ghirardi M, Montarolo PG, Kandel ER (1995) A novel intermediate stage in the transition between short- and long-term facilitation in the sensory to motor synapse of Aplysia. Neuron 14:413-420.

Grünbaum L, Müller U (1998) Induction of specific olfactory memory leads to a long-lasting activation of protein kinase $\mathrm{C}$ in the antennal lobe of the honeybee. J Neurosci 18:4384-4392. 
Herbert JM, Augereau JM, Gleye J, Maffrand JP (1990) Chelerythrine is a potent and specific inhibitor of protein kinase C. Biochem Biophys Res Commun 172:993-999.

Huber KM, Kayser MS, Bear MF (2000) Role for rapid dendritic protein synthesis in hippocampal mGluR-dependent long-term depression. Science 288:1254-1257.

Inoue M, Kishimoto A, Takai Y, Nishizuka Y (1977) Studies on a cyclic nucleotide-independent protein kinase and its proenzyme in mammalian tissues. II. Proenzyme and its activation by calcium-dependent proteases from rat brain. J Biol Chem 252:7610-7616.

Kang H, Welcher AA, Shelton D, Schuman EM (1997) Neurotrophins and time: different roles for TrkB signaling in hippocampal long-term potentiation. Neuron 19:653-664.

Kishimoto A, Kajikawa N, Shiota M, Nishizuka Y (1983) Proteolytic activation of calcium-activated, phospholipid-dependent protein kinase by calcium-dependent neutral protease. J Biol Chem 258:1156-1164.

Kobayashi E, Nakano H, Morimoto M, Tamaoki T (1989) Calphostin C (UCN-1028C), a novel microbial compound, is a highly potent and specific inhibitor of protein kinase C. Biochem Biophys Res Commun 159:548-553.

Levenson J, Endo S, Kategaya LS, Fernandez RI, Brabham DG, Chin J, Byrne JH, Eskin A (2000) Long-term regulation of neuronal high-affinity glutamate and glutamine uptake in Aplysia. Proc Natl Acad Sci USA $97: 12858-12863$.

Li XC, Giot JF, Kuhl D, Hen R, Kandel ER (1995) Cloning and characterization of two related serotonergic receptors from the brain and reproductive system of Aplysia that activate phospholipase C. J Neurosci 15:7585-7591.

Ling DSF, Benardo LS, Serrano PA, Blace N, Kelly MT, Crary JF, Sacktor TC (2002) Protein kinase $M \zeta$ is necessary and sufficient for LTP maintenance. Nat Neurosci 5:295-296.

Marinesco S, Carew TJ (2002) Serotonin release evoked by tail nerve stimulation in the CNS of Aplysia: characterization and relationship to heterosynaptic plasticity. J Neurosci 22:2299-2312.

Mauelshagen J, Parker GR, Carew TJ (1996) Dynamics of induction and expression of long-term synaptic facilitation in Aplysia. J Neurosci 16:7099-7108.

Müller U, Carew TJ (1998) Serotonin induces temporally and mechanistically distinct phases of persistent PKA activity in Aplysia sensory neurons. Neuron 21:1423-1434.

Oliet SH, Malenka RC, Nicoll RA (1997) Two distinct forms of long-term depression coexist in CA1 hippocampal pyramidal cells. Neuron 18:969-982.

Osten P, Valsamis L, Harris A, Sacktor TC (1996) Protein synthesisdependent formation of protein kinase $\mathrm{M} \zeta$ in long-term potentiation. J Neurosci 16:2444-2451.

Patterson SL, Pittenger C, Morozov A, Martin KC, Scanlin H, Drake C, Kandel ER (2001) Some forms of cAMP-mediated long-lasting potentiation are associated with release of BDNF and nuclear translocation of phospho-MAP kinase. Neuron 32:123-140.

Powell CM, Johnston D, Sweatt JD (1994) Autonomously active protein kinase $\mathrm{C}$ in the maintenance phase of $N$-methyl-D-aspartate receptorindependent long-term potentiation. J Biol Chem 269:27958-27963.

Sacktor TC, Schwartz JH (1990) Sensitizing stimuli cause translocation of protein kinase C in Aplysia sensory neurons. Proc Natl Acad Sci USA 87:2036-2039.

Sacktor TC, Osten P, Valsamis H, Jiang X, Naik MU, Sublette E (1993) Persistent activation of the zeta isoform of protein kinase $\mathrm{C}$ in the maintenance of long-term potentiation. Proc Natl Acad Sci USA 90:8310-8313.

Sato M, Tani E, Matsumoto T, Fujikawa H, Imajoh-Ohmi S (1997) Gener- ation of the catalytic fragment of protein kinase $\mathrm{C}$ alpha in spastic canine basilar artery. J Neurosurg 87:752-756.

Scholz KP, Byrne JH (1987) Long-term sensitization in Aplysia: biophysical correlates in tail sensory neurons. Science 235:685-687.

Sessoms JS, Chen SJ, Chetkovich DM, Powell CM, Roberson ED, Sweatt JD, Klann E (1992) $\mathrm{Ca}^{2+}$-induced persistent protein kinase $\mathrm{C}$ activation in rat hippocampal homogenates. Second Messengers Phosphoproteins 14:109-126.

Shea TB, Beermann ML, Griffin WR, Leli U (1994) Degradation of protein kinase $\mathrm{C}$ alpha and its free catalytic subunit, protein kinase $\mathrm{M}$, in intact human neuroblastoma cells and under cell-free conditions. Evidence that PKM is degraded by $\mathrm{mM}$ calpain-mediated proteolysis at a faster rate than PKC. FEBS Lett 350:223-229.

Sherff CM, Carew TJ (2002) Coincident induction of long-term facilitation at sensory-motor synapses in Aplysia: presynaptic and postsynaptic factors. Neurobiol Learn Mem 78:498-507.

Sossin WS (1997) An autonomous kinase generated during long-term facilitation in Aplysia is related to the $\mathrm{Ca}^{2+}$-independent protein kinase C Apl II. Learn Mem 3:389-401.

Sossin WS, Schwartz JH (1992) Selective activation of $\mathrm{Ca}^{2+}$-activated PKCs in Aplysia sensory neurons by 5-HT. J Neurosci 12:1160-1168.

Sossin WS, Sacktor TC, Schwartz JH (1994) Persistent activation of PKC during the development of long-term facilitation in Aplysia. Learn Mem 1:189-202.

Sutton MA, Carew TJ (2000) Parallel molecular pathways mediate expression of distinct forms of intermediate-term facilitation at tail sensorymotor synapses in Aplysia. Neuron 26:219-231.

Sutton MA, Masters SE, Bagnall MW, Carew TJ (2001) Molecular mechanisms underlying a unique intermediate phase of memory in Aplysia. Neuron 31:143-154.

Suzuki T, Okumura-Noji K, Ogura A, Tanaka R, Nakamura K, Kudo Y (1992) Calpain may produce a $\mathrm{Ca}^{2+}$-independent form of kinase $\mathrm{C}$ in long-term potentiation. Biochem Biophys Res Commun 189:1515-1520.

Toullec D, Pianetti P, Coste H, Bellevergue P, Grand-Perret T, Ajakane M, Baudet V, Boissin P, Boursier E, Loriolle F, Duhamel L, Charon D, Kirilovsky J (1991) The bisindolylmaleimide GF 109203X is a potent and selective inhibitor of protein kinase C. J Biol Chem 266:15771-15781.

Tully T, Preat T, Boynton SC, Del Vecchio M (1994) Genetic dissection of consolidated memory in Drosophila. Cell 79:35-47.

Vanderklish P, Saido TC, Gall C, Arai A, Lynch G (1995) Proteolysis of spectrin by calpain accompanies theta-burst stimulation in cultured hippocampal slices. Brain Res Mol Brain Res 32:25-35.

Vanderklish P, Bednarski E, Lynch G (1996) Translational suppression of calpain blocks long-term potentiation. Learn Mem 3:209-217.

Walters ET (1987) Multiple sensory neuronal correlates of site-specific sensitization in Aplysia. J Neurosci 7:408-417.

Walters ET, Byrne JH, Carew TJ, Kandel ER (1983a) Mechanoafferent neurons innervating tail of Aplysia. I. Response properties and synaptic connections. J Neurophysiol 50:1522-1542.

Walters ET, Byrne JH, Carew TJ, Kandel ER (1983b) Mechanoafferent neurons innervating tail of Aplysia. II. Modulation by sensitizing stimulation. J Neurophysiol 50:1543-1559.

Yanow SK, Manseau F, Hislop J, Castellucci VF, Sossin WS (1998) Biochemical pathways by which serotonin regulates translation in the nervous system of Aplysia. J Neurochem 70:572-583.

Zhang F, Endo S, Cleary LJ, Eskin A, Byrne JH (1997) Role of transforming growth factor-beta in long-term synaptic facilitation in Aplysia. Science 275:1318-1320.

Zhang ZS, Fang B, Marshak DW, Byrne JH, Cleary LJ (1991) Serotonergic varicosities make synaptic contacts with pleural sensory neurons of Aplysia. J Comp Neurol 311:59-270. 